\title{
The geometry of PSR B0031-07
}

\author{
J. M. Smits ${ }^{1}$, D. Mitra ${ }^{2}$, B. W. Stappers ${ }^{3,4}$, J. Kuijpers ${ }^{1}$, P. Weltevrede ${ }^{4}$, A. Jessner ${ }^{5}$, and Y. Gupta ${ }^{2}$ \\ 1 Department of Astrophysics, Radboud University, Nijmegen, The Netherlands \\ e-mail: Roy.Smits@manchester.ac.uk \\ 2 National Center for Radio Astrophysics, Pune, India \\ ASTRON, Dwingeloo, The Netherlands \\ 4 Astronomical Institute "Anton Pannekoek", Amsterdam, The Netherlands \\ 5 Max-Planck-Insitut für Radioastronomy, Bonn, Germany
}

Received 23 August 2006 / Accepted 20 January 2007

\begin{abstract}
Context. PSR B0031-07 is well known to exhibit three different modes of drifting sub-pulses (mode A, B and C). It has recently been shown that in a multifrequency observation, consisting of 2700 pulses, all driftmodes were visible at low frequencies, while at $4.85 \mathrm{GHz}$ only mode-A drift or non-drifting emission was detected. This suggests that modes $\mathrm{A}$ and $\mathrm{B}$ are emitted in sub-beams, rotating at a fixed distance from the magnetic axis, with the mode-B sub-beams being closer to the magnetic axis than the mode-A sub-beams. Diffuse emission between the sub-beams can account for the non-drifting emission.

Aims. Using the results of an analysis of simultaneous multifrequency observations of PSR B0031-07, we set out to construct a geometrical model that includes emission from both sub-beams and diffuse emission and describes the regions of the radio emission of PSR B0031-07 at each emission frequency for driftmodes A and B.

Methods. Based on the vertical spacing between driftbands, we have determined the driftmode of each sequence of drift. To restrict the model, we calculated average polarisation and intensity characteristics for each driftmode and at each frequency.

Results. The model reproduces the observed polarisation and intensity characteristics, suggesting that diffuse emission plays an important role in the emission properties of PSR B0031-07. The model further suggests that the emission heights of this pulsar range from a few kilometers to a little over 10 kilometers above the pulsar surface. We also find that the relationships between height and frequency of emission that follow from curvature radiation and from plasma-frequency emission could not be used to reproduce the observed frequency dependence of the width of the average intensity profiles.
\end{abstract}

Key words. stars: neutron - stars: pulsars: general - stars: pulsars: individual: PSR B0031-07

\section{Introduction}

Pulsar B0031-07 is well known for its three modes of drifting sub-pulses. They are called mode A, B and C and are characterised by their values for $P_{3}$ of 12,7 and 4 times the pulsar period, respectively (Huguenin et al. 1970). This pulsar has been thoroughly studied at low observing frequencies (Huguenin et al. 1970; Krishnamohan 1980; Wright 1981; Vivekanand 1995; Vivekanand \& Joshi 1997, 1999; Joshi \& Vivekanand 2000), but only rarely at an observing frequency above $1 \mathrm{GHz}$ (Wright \& Fowler 1981; Kuzmin et al. 1986; Izvekova et al. 1993). Recently, Smits et al. (2005) analysed simultaneous multifrequency observations from both the Westerbork Synthesis Radio Telescope and the Effelsberg Radio Telescope and detected all three drift modes at $325 \mathrm{MHz}$, but only detected drift mode A at $4.85 \mathrm{GHz}$. The pulses that were classified as mode B or C at low frequency only showed non-drifting emission at high frequency. On the basis of their findings, they suggest a geometrical model where modes A and B at a given frequency are emitted in two concentric rings around the magnetic axis with mode $\mathrm{B}$ being nested inside mode $\mathrm{A}$. This nested configuration is preserved across frequency with the higher frequency arising closer to the stellar surface compared to the lower one, consistent with the well known radius-to-frequency mapping operating in pulsars. Due to the rare occurrence of mode $\mathrm{C}$, they did not attempt to include this drift mode in their model.
Here we analyse new multifrequency observations of PSR B0031-07, obtained with the Giant Metrewave Radio Telescope, the Westerbork Synthesis Radio Telescope and the Effelsberg Radio Telescope simultaneously. In total, the observations contain 136000 pulses spread over 7 different frequencies. From these observations we attempt to restrict the geometry of this pulsar and create a model that reproduces a great number of its observed characteristics.

In Sect. 2 we explain how the observations have been obtained, how the different modes of drift have been determined, and which further analyses have been carried out. In Sect. 3 we present our results, which is followed by the modelling of the geometry of the emission of PSR B0031-07 in Sect. 4. The discussion and conclusions follow in Sects. 5 and 6, respectively.

\section{Method}

\subsection{Definitions}

To describe the observational drift of sub-pulses we use three parameters, which are defined as follows: $P_{3}$ is the spacing, at the same pulse phase, between drift bands in units of pulsar periods $\left(P_{1}\right)$; this is the "vertical" spacing when the individual radio profiles obtained during one stellar rotation are plotted one above the other (stacked). $P_{2}$ is the interval between successive sub-pulses within the same pulse, given in degrees, and $\Delta \phi$, the sub-pulse phase drift, is the fraction of pulse period over which 
Table 1. List of known parameters of PSR B0031-07. All values are from Taylor et al. (1993).

\begin{tabular}{ll}
\hline \hline Parameter & Value \\
\hline$P_{1}$ & $0.94295 \mathrm{~s}$ \\
$\dot{P}$ & $4.083 \times 10^{-16}$ \\
$\mathrm{DM}$ & $10.89 \mathrm{pc} \mathrm{cm}^{-3}$ \\
$S_{400}$ & $95 \mathrm{mJy}$ \\
$S_{1400}$ & $11 \mathrm{mJy}$ \\
$B_{\text {surf }}$ & $6.31 \times 10^{11} \mathrm{G}$ \\
$\dot{E}$ & $1.9 \times 10^{31} \mathrm{erg} / \mathrm{s}$ \\
\hline
\end{tabular}

a sub-pulse drifts in one pulse period, given in ${ }^{\circ} / P_{1}$. Note that $P_{2}=P_{3} \times \Delta \phi$.

These parameters are often thought to be associated with beams of emission (sub-beams), rotating at a fixed distance around the magnetic axis. When each sub-pulse that is seen to be drifting in consecutive pulses (which is observed as a drift band) is due to emission from an individual sub-beam, then $P_{3}$ is the rotation time of this configuration divided by the number of sub-beams. However, when the sub-beams rotate fast enough, it becomes possible that multiple sub-beams contribute to one drift band. This is called aliasing and is hard to detect. The observed $P_{3}$ is then no longer the rotation time divided by the number of sub-beams, but depends on the degree of aliasing.

\subsection{Observations}

Here we present observations of PSR B0031-07, which were obtained with the Giant Metrewave Radio Telescope (GMRT), the Westerbork Synthesis Radio Telescope (WSRT) and the Effelsberg Radio Telescope (EFF). They comprise 7 different frequencies. A great part of the observations are simultaneous at different frequencies and include $4 \mathrm{~h}$ in which radio emission from PSR B0031-07 is observed at 5 frequencies simultaneously.

The 150, 243, 325, 610 and $1167 \mathrm{MHz}$ observations were conducted using the GMRT located in Pune, India. The GMRT is a multi-element aperture synthesis telescope (Swarup et al. 1991) consisting of 30 antennas distributed over a $25-\mathrm{km}$ diameter area which can be configured as a single dish (Gupta et al. 2000). The antennas each have a gain of $0.3 \mathrm{~K} / \mathrm{Jy}$. The system temperature for the different frequencies are $T_{\text {sys }}^{157}=482 \mathrm{~K}$, $T_{\text {sys }}^{243}=177 \mathrm{~K}, T_{\text {sys }}^{325}=108 \mathrm{~K}, T_{\text {sys }}^{607}=92 \mathrm{~K}$ and $T_{\text {sys }}^{1167}=76 \mathrm{~K}$. The signals from the antennas at 150,243, 325 and $610 \mathrm{MHz}$ have circular polarisation, while at $1167 \mathrm{MHz}$ the signals are linearly polarised. At any frequency, orthogonally polarised complex voltages arrive at the sampler from each of the antennas. The voltage signals are subsequently sampled at the Nyquist rate and processed through a digital receiver system consisting of a correlator, the GMRT array combiner (GAC) and a pulsar back-end for GAC. The signals selected by the user are added in phase and fed to the pulsar back-end. The pulsar back-end computes both the auto- and cross-polarised power, which was then recorded at a sampling rate of $0.512 \mathrm{~ms}$. We have carried out simultaneous observation at 243, 607 and $1167 \mathrm{MHz}$ by splitting the whole array in to 3 different sub-arrays. We have used a scheme wherein the digital sub-array combiner, which does the incoherent addition of the multi-channel baseband data from different antennas, is programmed to blank the data for a selected set of frequency channels for antennas from a given sub-array. This is akin to setting non-overlapping filter banks for each subarray and allows the $16 \mathrm{MHz}$ bandwidth to be divided between the different radio frequency bands of observations, without any overlap between signals from the different bands, while still preserving the time alignment of the data from the different frequency bands. The 243 and $610 \mathrm{MHz}$ observations involved polarimetry and the observations have been corrected for Faraday rotation, dispersion and parallactic angle. Variations in the rotation measure, due to intrinsic fluctuations of the ionosphere during the length of one observation causes a deviation in the position angle of less than $5^{\circ}$ at an observation frequency of $243 \mathrm{MHz}$. Towards higher frequencies, this deviation decreases with $\lambda^{2}$. To correct for an instrumental polarisation effect, the polarisation calibrator PSR B1929+10 was observed at several parallactic angles, and was used to calibrate the data using the technique described by Mitra et al. (2005). In most cases the corruption due to leakage was found to be small, and could be corrected up to an accuracy of 5\%.

The WSRT observations were made at a frequency of $840 \mathrm{MHz}$ with a bandwidth of $80 \mathrm{MHz}$ and recorded using the pulsar back-end, PuMa (Voûte et al. 2002). The signals from 14 telescopes were added with appropriate delays resulting in a gain of $1.2 \mathrm{~K} / \mathrm{Jy}$. The system temperature at this frequency is around $T_{\text {sys }}=150 \mathrm{~K}$. The Effelsberg observations were made at a frequency of $4.85 \mathrm{GHz}$ and a bandwidth of $500 \mathrm{MHz}$. The gain is $1.5 \mathrm{~K} / \mathrm{Jy}$ and the system temperature at this frequency is $T_{\text {sys }}=27 \mathrm{~K}$. The polarisation calibration is described in chapter 4 of Hoensbroech (1999). The WSRT observations have been corrected for Faraday rotation, dispersion and for any instrumental polarisation effects using a procedure described in the Appendix of Edwards \& Stappers (2004).

A $50-\mathrm{Hz}$ signal present in the Effelsberg observation has been removed by Fourier transforming the entire sequence, removing the $50 \mathrm{~Hz}$ peak and Fourier transforming back. Table 2 lists the details of all the observations. All the observations were aligned by correlating long sequences of pulses with pulses from the observation at $607 \mathrm{MHz}$ that were obtained simultaneously. This procedure removes any delay in arrival time between pulses at different frequencies due to retardation and aberration. The accuracy of this alignment is within $1 \mathrm{~ms}$.

\subsection{Finding the drift sequences}

Smits et al. (2005) have shown that for at least one of the drift modes the value for $P_{3}$ is the same for both high and low frequency observations. This is consistent with the concept that the emission of pulsars originates from sub-beams of particles that rotate around the magnetic axis due to the force-free motion of the particles in the strong magnetic field. Here, we have investigated the values of $P_{3}$ for both mode $\mathrm{A}$ and mode $\mathrm{B}$ over a larger frequency range, using the same method as described by Smits et al. (2005). From the observations we chose 1500 consecutive pulses for which the sub-pulses were visible in at least 4 frequencies. We then tested for each drift sequence whether the value of $P_{3}$ was the same at each frequency. We can confirm that in these 1500 pulses, within errors, $P_{3}$ remains constant for mode A over the frequencies $243,607,840 \mathrm{MHz}$ and $4.85 \mathrm{GHz}$. Furthermore, we unexpectedly found multiple detections of mode-B drift at $4.85 \mathrm{GHz}$, whereas Smits et al. (2005) found no mode-B drift in 2700 pulses at $4.85 \mathrm{GHz}$, nor in 5350 pulses at an even lower frequency of $1.41 \mathrm{GHz}$. Still, we could not accurately measure $P_{3}$ at $4.85 \mathrm{GHz}$ when the pulsar was in mode $\mathrm{B}$. We did confirm that in this mode $P_{3}$ remains constant over the frequencies 243, 607 and $840 \mathrm{MHz}$. We then made the assumption that there is indeed one fundamental period associated with the vertical spacing between drift bands, such as 
Table 2. Details of the observations of PSR B0031-07.

\begin{tabular}{lllllll}
\hline \hline Date & $\begin{array}{l}\text { Start time (UT) } \\
\text { (s after midnight) }\end{array}$ & Telescope & $\begin{array}{l}\text { Freq. } \\
\text { (MHz) }\end{array}$ & $\begin{array}{l}\text { Time res. } \\
(\mathrm{ms})\end{array}$ & $\begin{array}{l}\text { Bandwidth } \\
(\mathrm{MHz})\end{array}$ & $\begin{array}{l}\text { Number of } \\
\text { pulses }\end{array}$ \\
\hline $02-09-2004$ & 62616 & GMRT & 325 & 0.512 & 16 & 2678 \\
$03-09-2004$ & 69092 & GMRT & 243 & 0.512 & 6.25 & 14607 \\
$03-09-2004$ & 69092 & GMRT & 607 & 0.512 & 9.75 & 14607 \\
$03-09-2004$ & 72150 & WSRT & 840 & 0.8192 & 80 & 16140 \\
$03-09-2004$ & 75847 & EFF & 4850 & 0.9206 & 500 & 31042 \\
$03-09-2004$ & 80087 & GMRT & 1167 & 1.024 & 16 & 10145 \\
$04-09-2004$ & 00554 & GMRT & 243 & 1.024 & 6.25 & 4409 \\
$04-09-2004$ & 00554 & GMRT & 607 & 1.024 & 9.75 & 4409 \\
$07-09-2004$ & 69522 & GMRT & 607 & 0.512 & 9.75 & 4718 \\
$07-09-2004$ & 71441 & WSRT & 840 & 0.8192 & 80 & 16781 \\
$07-09-2004$ & 73690 & GMRT & 243 & 0.512 & 6.25 & 14394 \\
$24-08-2005$ & 43212 & GMRT & 157 & 0.512 & 16 & 2229 \\
\hline
\end{tabular}

the rotation speed of sub-beams around the magnetic axis, that is the same at each frequency at each pulse phase. With this assumption, we determined the drift mode of each pulse for all of the observations. This was achieved by visually finding drift sequences at the frequency that showed this drift most clearly. Whenever possible, these sequences were then inspected at the remaining available frequencies to improve the exact beginning and end of the sequences. Finally, $P_{3}$ was calculated for each sequence and classified as either mode A, B, or C. Even though the time span of the observations is about $10 \mathrm{~h}$, we only detected 4 short drift sequences with a mode-C drift, none of them were detected at $4.85 \mathrm{GHz}$. Because of the low number of pulses which were in a mode-C drift, we limited the analysis to sequences of pulses which were in mode-A or mode-B drift.

\subsection{Average polarisation profiles}

Once the drift class of all the sequences was known, all pulses in a drift class were averaged together for each frequency separately. This resulted in average mode- $\mathrm{A}$ and $\mathrm{B}$ polarisation profiles and position angle sweeps at each frequency. We also measured the widths of each of these profiles at $10 \%$ of the peak values.

\subsection{Frequency dependence of the fractional drift intensity}

Smits et al. (2005) have suggested that the mode-A and mode-B emission originate from different regions of the magnetosphere. This means that the line of sight intersection with the sub-beams is different for both drift modes (see their Figs. 4 and 5). In particular, this causes the line of sight to miss the center of the ring of mode-B sub-beams at high frequency and only cuts through a diffuse component surrounding the sub-beams. As a result we do not see any drift bands at high frequency whenever the lowfrequency emission reveals a mode-B drift. If the line of sight intersection with the sub-beams indeed determines how clearly a drift pattern can be observed, then the drift pattern can provide information about the geometry of the sub-beams. To measure how far the line of sight intersection is away from the center of the sub-beams in a drift sequence, we calculated the fractional drift intensity as follows. First we took the amplitude at each pulse phase of each pulse in the sequence and stacked them on top of each other to produce a 2D time series of the same type as shown in Fig. 5. We then calculated the absolute values of the Fourier transform at each pulsar phase of this 2D time series. The resulting Longitude-Resolved Fluctuation Spectrum (LRFS) was then averaged over a pulse phase window

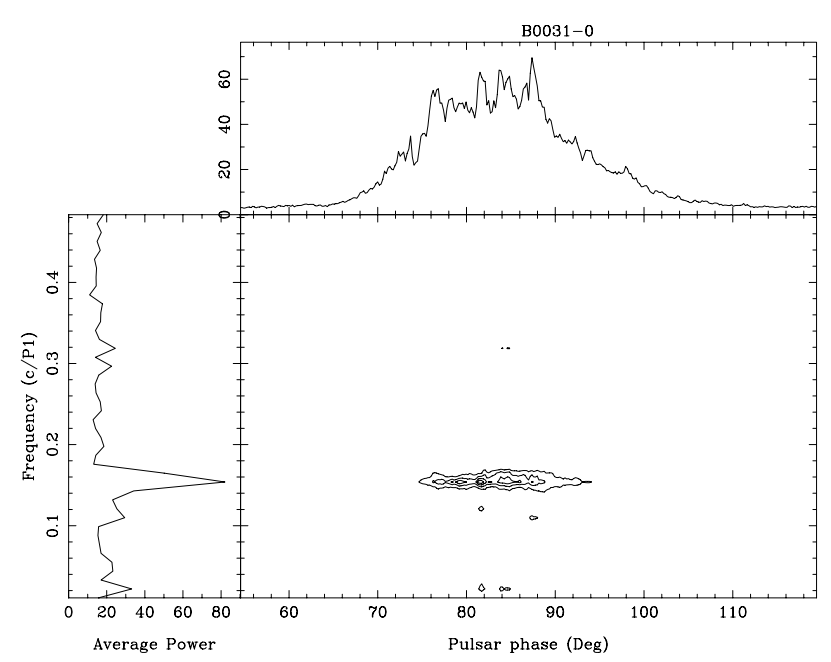

Fig. 1. Contour plot of the LRFS of PSR B0031-07 at $0.325 \mathrm{GHz}$ as a function of pulse phase during a sequence of mode-B drift. The left panel shows the power spectrum averaged over phase. The upper panel shows the power averaged over frequency.

containing the pulsar on pulse, giving a Longitude-Averaged Fluctuation Spectrum (LAFS). A contour plot of a LRFS, as well as the LAFS, is shown in Fig. 1. The drift intensity was calculated by integrating the LAFS over a small frequency range containing the reciprocal of the $P_{3}$ value of the sequence and then subtracting the integration of the LAFS over a small frequency range containing no periodicities. By dividing the drift intensity by the total intensity in the LAFS of the pulsar signal we obtained the fractional drift intensity. This fractional drift intensity was calculated for all sequences of drift at each observed frequency. When the line of sight starts to miss the center of the ring of sub-beams and only cut through diffuse emission, then the fractional drift intensity should become low.

\subsection{Width of average drift profiles}

The geometrical model that will be constructed to describe the single pulse emission from PSR B0031-07 consists of both drifting and non-drifting emission. From the model we can calculate at each frequency and for each drift mode the width of the average profile resulting from the drifting emission only. In order to use this width to test how well the model describes the observed emission, we needed to determine the width of 
the average profiles resulting from the drifting emission only, in the observations. This was done for each frequency as follows. For each drift sequence, we determined the average drift profile by integrating the power in the LRFS at each pulse phase over a small frequency range, containing the reciprocal of the $P_{3}$ value of the sequence. For each drift mode these profiles were averaged together and their widths were measured.

\subsection{Frequency dependence of $P_{2}$}

The change of $P_{2}$ with observation frequency reflects the change of the size of the emission zone with respect to the total path of the line of sight intersection at different heights above the pulsar surface. It therefore limits the radius-to-frequency mapping that can be used for any model. $P_{2}$ is often calculated by averaging autocorrelations of single pulses, containing more than one subpulse. For PSR B0031-07 this method fails at high frequencies where there are no pulses containing more than one sub-pulse. We therefore calculated $P_{2}$, for each drift sequence, as the product of $P_{3}$ and the sub-pulse phase drift $(\Delta \phi)$ of that sequence. The phase drift was measured by cross-correlating consecutive pulses of each drift sequence and averaging the resulting crosscorrelations. The peaks of the average cross-correlations were fitted with a quadratic polynomial, of which the phase shift of the maximum was taken as the phase drift. For each drift mode and observation frequency the values for $P_{2}$ were averaged over many drift sequences to obtain the frequency dependence of $P_{2}$ for each drift mode. The values for $P_{2}$ are not affected by a possible aliasing.

\section{Results}

The (polarisation) profiles of PSR B0031-07 for 7 frequencies are shown in Fig. 2. No polarisation was recorded for the 157, 325 and $1167 \mathrm{MHz}$ observations. The 157 and $325 \mathrm{MHz}$ observations were not simultaneous with the other observations and were aligned to have the center of the profiles at the same pulse phase as the center of the 243 and $607 \mathrm{MHz}$ profiles, respectively. The widths at $10 \%$ of the maximum were measured for all the intensity profiles. They are shown in Table 3 . The effects of dispersion are negligible and have no significant contribution to the measured width. The errors quoted here and elsewhere in the paper are 1-sigma errors.

Figure 3 shows the fractional drift intensity in drift sequences for 5 different frequencies over a duration of $4 \mathrm{~h}$ and $15 \mathrm{~min}$. Due to interference the drift intensity could not be determined in some parts of the observations. These parts and parts where no pulses were observed are marked in Fig. 3 as hatched areas. The average fractional drift intensities of drift modes A and B for four of the frequencies are listed in Table 4. The errors were estimated by using the rms of the region in the LAFS that does not contain the reciprocal of the $P_{3}$ value. The $1167 \mathrm{MHz}$ observation is not listed because this observation does not contain enough drift sequences to give a reliable average value. The widths of the average drift profiles, i.e. of only the drifting component, for each drift mode at each frequency are shown in Table 5. At $4.85 \mathrm{GHz}$ there was not enough signal in the mode-B profile to measure a width. Table 6 lists the average values of $P_{2}$ for drift modes $\mathrm{A}$ and $\mathrm{B}$ for 7 different observation frequencies. Note that the observations at 157 and $325 \mathrm{MHz}$ were not part of the simultaneous observations.

\section{Modelling the geometry}

Here, we set out to find a geometry that can reproduce a great number of the observed features of this pulsar, which includes the position angle sweep (without the orthogonal mode jumps) and the frequency dependences of the width of the average intensity profile, the width of the average drift profile, the fractional drift intensity and $P_{2}$, for drift modes $\mathrm{A}$ and $\mathrm{B}$.

We first try to limit the geometrical possibilities by fitting the widths of the observed average profiles to three different models that restrict the relationship between height and frequency of emission. All models assume that the intensity of the radio emission decreases with distance from the maximally emitting ring of field lines as a Gaussian given by

$I=\exp \left(-\frac{1}{2}\left(\frac{\chi-\chi_{0}}{\chi_{\mathrm{w}}}\right)^{2}\right)$

where $I$ is the intensity, $\chi$ is the angle between the magnetic axis and the foot point on the surface of the star of the field line from which emission can be observed (see Fig. 4), $\chi_{0}$ is the angle between the magnetic axis and the foot point on the surface of the star of the maximally emitting field line and $\chi_{\mathrm{w}}$ is the angle between the foot points on the surface of the star of the two field lines for which the intensity has dropped to $\mathrm{e}^{-\frac{1}{2}}$ times the maximum intensity.

In the first model, the curvature radiation model, we assume that the frequency of emission is equal to the characteristic frequency for curvature radiation, given by (Jackson 1999, Eq. (14.81))

$\omega=\frac{3}{2} \gamma^{3}\left(\frac{c}{r_{\mathrm{cr}}}\right)$,

where $\gamma$ is the Lorentz factor of the secondary plasma, $c$ is the speed of light and $r_{\mathrm{cr}}$ is the radius of curvature at the emission point. Following the derivation from Gangadhara (2004), the dipolar geometry of the magnetic field dictates how the radius of curvature is related to the distance between the emission point and the center of the star, $r$, and the polar angle to the magnetic axis, $\theta$, as

$r_{\mathrm{cr}}=\frac{r}{\sin \theta} \frac{[5+3 \cos (2 \theta)]^{\frac{3}{2}}}{3 \sqrt{2}[3+\cos (2 \theta)]}$

For angles smaller than $10^{\circ}$ this can be linearized within an error of $1.5 \%$ to give

$r_{\mathrm{cr}}=\frac{4}{3} r / \theta$

In the second model, the plasma-frequency model, we assume that the radiation is emitted at the local relativistic plasma frequency, given in SI by

$\omega_{\mathrm{rp}}^{2}=\langle\gamma\rangle^{-1} \frac{e^{2} n}{\epsilon_{0} m_{\mathrm{e}}}$

where $n$ is the sum of electron and positron density and $e$ and $m_{\mathrm{e}}$ are the electron charge and mass. We further assume that there is a distinct region around the magnetic axis where shots of particles can create a secondary pair plasma with a fixed particle density which only depends on altitude. The frequency of occurrence of these shots is assumed to decrease as a Gaussian with increasing distance from the maximum intensity field lines. Thus, the intensity of the emission falls off with distance from 

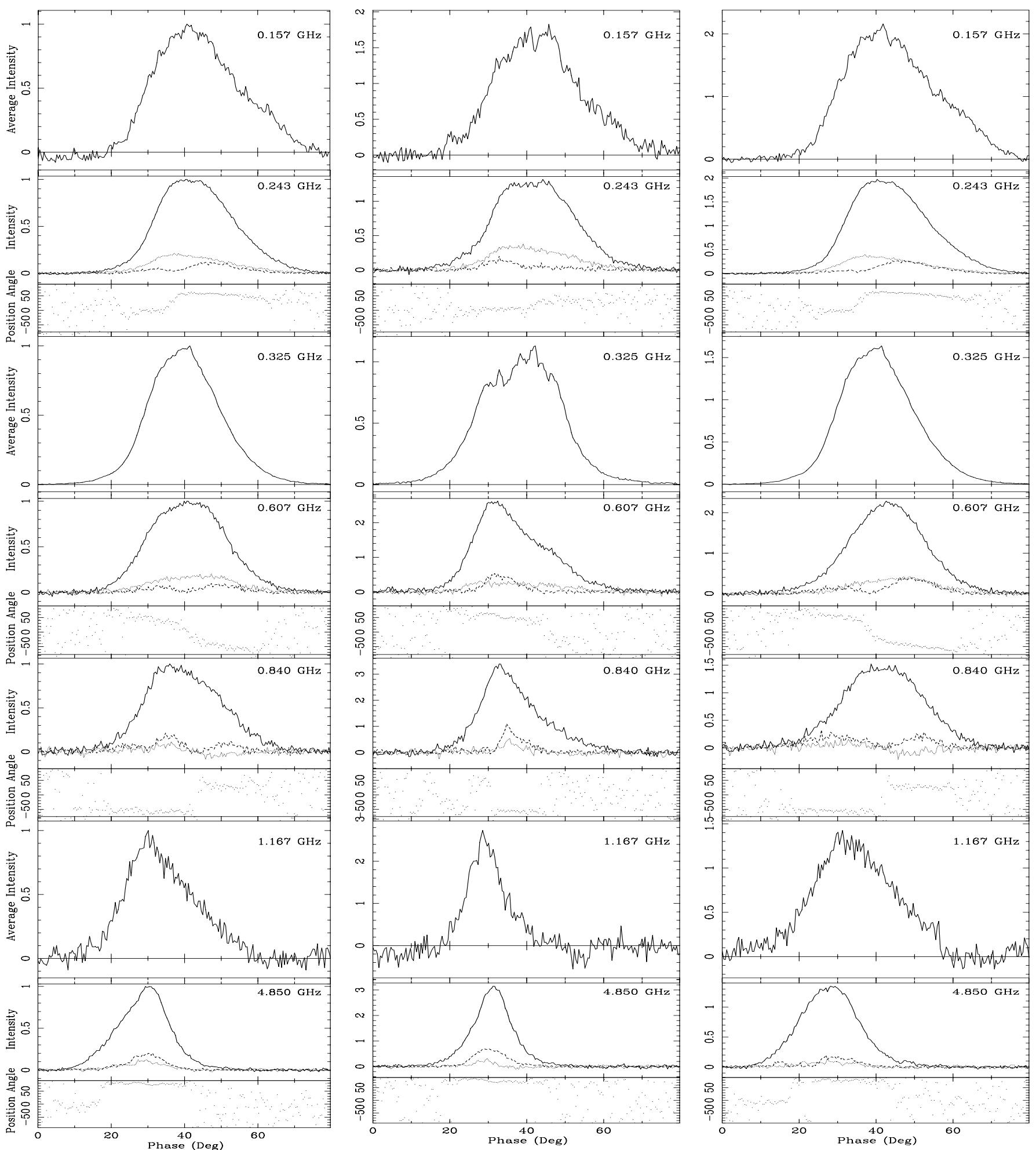

Fig. 2. Average polarisation profiles from all the observations. From left to right are the average profiles of all pulses, of pulses that are in mode A and of pulses that are in mode B. The solid line is the intensity and the dashed and dotted lines are the linear and circular polarisation, respectively. Only the 243, 607, 840, 1167 and $4850 \mathrm{MHz}$ profiles were obtained simultaneously. All profiles are binned to the lowest time resolution of $1.024 \mathrm{~ms}$. The method for alignment is described in Sect. 2.2.

the maximally emitting field lines, while the particle density of the shots and the frequency of emission remains constant at constant emission height. Since, along the open field lines, the density of relativistically moving secondary pair plasma falls off approximately with the cube of the distance to the center of the star, in a dipolar field, on account of flux conservation, the 

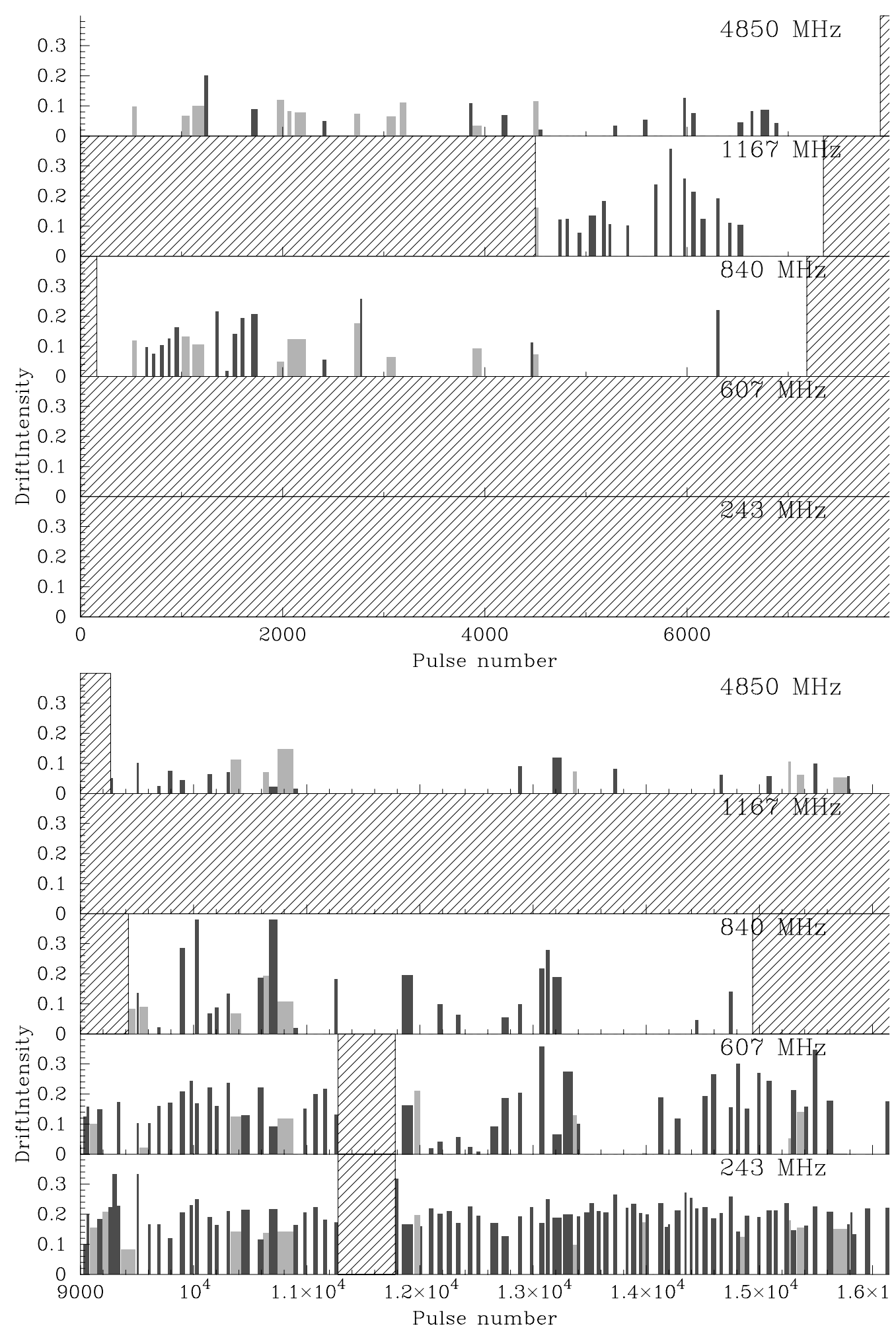

Fig. 3. Fractional drift intensity in drift sequences at 5 different frequencies. The gray columns represent sequences of pulses for which a drift intensity could be detected. Light-gray indicates mode-A drift, whereas dark-gray indicates mode-B drift. The hatched areas indicate regions during which no pulses were observed, or the quality of the observation was not good enough to determine the drift intensity. The lower graph is the continuation of the upper graph. The total duration is $4 \mathrm{~h}$ and $15 \mathrm{~min}$.

frequency drops with distance to the power $\frac{3}{2}$. This leads to the following relationship between the altitude and frequency of emission (Ruderman \& Sutherland 1975):

$r=\left(\frac{\omega}{\omega_{0}}\right)^{-\frac{2}{3}}$.
Finally, we try a version of an empirical relationship, as given by Thorsett (1991):

(6) $r=h_{0}\left(1+\left(\frac{\omega}{\omega_{0}}\right)^{-\frac{2}{3}}\right)$, 
Table 3. The $10 \%$-widths of the average profiles from all pulses and from the drift modes A and B from the observations of PSR B0031-07 at 7 different frequencies.

\begin{tabular}{lccc}
\hline \hline $\begin{array}{l}\text { Frequency } \\
(\mathrm{MHz})\end{array}$ & Width (deg) & $\begin{array}{c}\text { Width mode A } \\
(\mathrm{deg})\end{array}$ & $\begin{array}{c}\text { Width mode B } \\
(\mathrm{deg})\end{array}$ \\
\hline 157 & $45.7 \pm 1.8$ & $44 \pm 5$ & $46.3 \pm 1.4$ \\
243 & $42.3 \pm 0.6$ & $39.5 \pm 1.5$ & $42.3 \pm 0.6$ \\
325 & $40.6 \pm 0.15$ & $39.4 \pm 0.3$ & $40.30 \pm 0.12$ \\
607 & $39.8 \pm 1.1$ & $32 \pm 4$ & $39.3 \pm 0.9$ \\
840 & $40.9 \pm 0.6$ & $32.2 \pm 0.8$ & $43.0 \pm 0.9$ \\
1167 & $36 \pm 3$ & $25 \pm 6$ & $38 \pm 5$ \\
4850 & $33.7 \pm 0.8$ & $23.6 \pm 0.6$ & $32.8 \pm 0.9$ \\
\hline
\end{tabular}

Table 4. Average fractional drift intensity of drift mode A and B for four different frequencies.

\begin{tabular}{lcc}
\hline \hline $\begin{array}{l}\text { Frequency } \\
(\mathrm{MHz})\end{array}$ & $\begin{array}{c}\text { Fractional } \\
\text { drift intensity } \\
\text { in mode A }\end{array}$ & $\begin{array}{c}\text { Fractional } \\
\text { drift intensity } \\
\text { in mode B }\end{array}$ \\
\hline 243 & $0.14 \pm 0.05$ & $0.20 \pm 0.05$ \\
607 & $0.07 \pm 0.02$ & $0.11 \pm 0.03$ \\
840 & $0.07 \pm 0.02$ & $0.04 \pm 0.02$ \\
4850 & $0.07 \pm 0.02$ & $0.02 \pm 0.01$ \\
\hline
\end{tabular}

which is similar to the plasma-frequency model, but has an extra distance parameter $h_{0}$ that allows for a minimum emission height. For all three models we simulated the intensity profile at 7 different frequencies, corresponding to the 7 frequencies of the observations, for different values of the parameters of the models. These parameters and the limits within which they were varied, are listed in Table 7. $\gamma$ and $\omega_{0}$ were varied logarithmically, the other parameters linearly. We then calculated the widths at $10 \%$ of the maximum. From these widths and the widths from the mode-A and mode-B intensity profiles from the observations we calculated the reduced chi-square for each set of parameters for each drift mode. We then minimized these chisquares to fit the values for the parameters. Initial values for $\alpha$ and $\beta$ were found by fitting the rotating vector model from Radhakrishnan \& Cooke (Radhakrishnan \& Cooke 1969) to the position angle from all the observations for which polarisation was recorded. As can be seen in the panels of Fig. 2, the positionangle sweep is very straight, apart from orthogonal mode jumps which causes differences in the position-angle sweep at different frequencies. After excluding the pulsar phases that contain an orthogonal mode jump, each sweep can be reproduced with the rotating vector model from Radhakrishnan \& Cooke, when $\alpha$ and $\beta$ are kept equal and in the range of $0.1^{\circ}$ to $6^{\circ}$.

Once an initial relationship between the altitude and frequency of emission was found, we proceeded to include both diffuse emission and emission from rotating sub-beams to produce single pulses in two different drift modes for a number of frequencies. The parameters of this model were then fitted to reproduce the position angle sweep and the frequency dependences of the width of the average intensity profile, the width of the average drift profile, the fractional drift intensity and $P_{2}$, for drift modes A and B. Also, we used the result from Smits et al. (2005) that shows that for drift mode A at low frequencies, the line of sight intersection just penetrates the maximally emitting field lines, causing the observed double component in the upper-left panel of their Fig. 8.
Table 5. The 10\%-widths of the average drift profiles for drift mode A and B for 7 different frequencies. A "-" indicates that there was not enough signal to measure a width.

\begin{tabular}{lcc}
\hline \hline $\begin{array}{l}\text { Frequency } \\
\text { (MHz) }\end{array}$ & $\begin{array}{c}\text { 10\%-width of } \\
\text { mode A (deg) }\end{array}$ & $\begin{array}{c}\text { 10\% width of } \\
\text { mode B (deg) }\end{array}$ \\
\hline 157 & $28 \pm 2$ & $35.0 \pm 1.5$ \\
243 & $27 \pm 5$ & $35.2 \pm 0.9$ \\
325 & $33.4 \pm 0.4$ & $35.6 \pm 0.4$ \\
607 & $27 \pm 3$ & $30 \pm 4$ \\
840 & $12.2 \pm 0.9$ & $10 \pm 4$ \\
1167 & $9 \pm 3$ & $3.1 \pm 1.1$ \\
4850 & $16.4 \pm 0.9$ & - \\
\hline
\end{tabular}

Table 6. The average values of $P_{2}$ for drift modes A and B for 7 different frequencies. A "-" indicates that there was not enough signal to measure $P_{2}$.

\begin{tabular}{lcc}
\hline \hline $\begin{array}{l}\text { Frequency } \\
(\mathrm{MHz})\end{array}$ & $\begin{array}{c}P_{2} \text { of mode A } \\
(\mathrm{deg})\end{array}$ & $\begin{array}{c}P_{2} \text { of mode B } \\
(\mathrm{deg})\end{array}$ \\
\hline 157 & $18.9 \pm 1.2$ & $19.2 \pm 0.7$ \\
243 & $24 \pm 4$ & $18.7 \pm 1.6$ \\
325 & $19.8 \pm 1.0$ & $18.7 \pm 0.6$ \\
607 & $21 \pm 3$ & $18.7 \pm 0.6$ \\
840 & $10 \pm 3$ & $14.0 \pm 1.9$ \\
1167 & $14 \pm 5$ & $12 \pm 3$ \\
4850 & $14 \pm 2$ & - \\
\hline
\end{tabular}

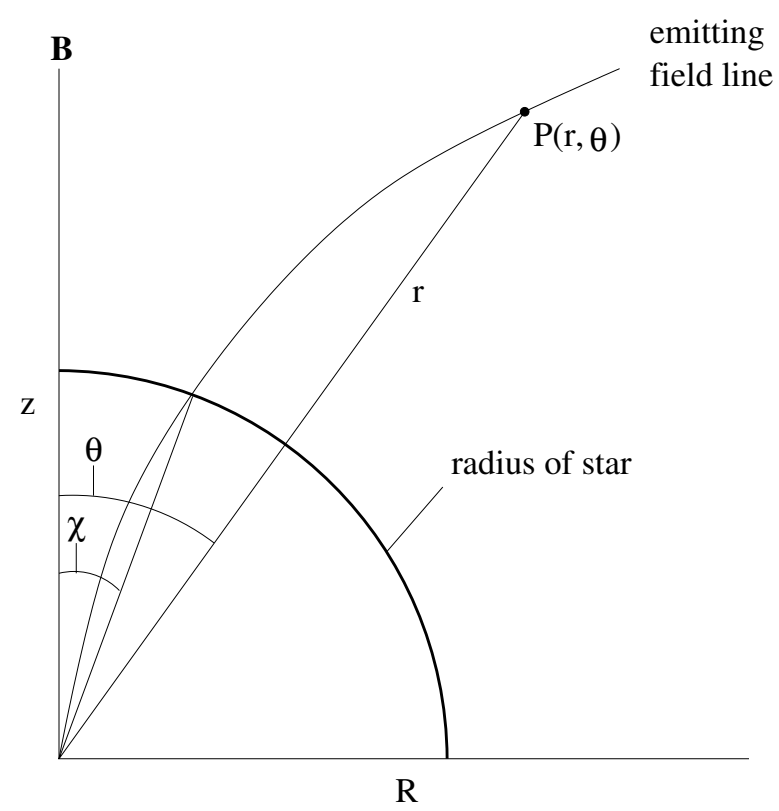

Fig. 4. Sketch of a field line as a function of distance along the magnetic axis $(z)$ and distance from the magnetic axis $(R)$ near the pulsar, to show the definition of the angle $\chi$. Point $P$ on an emitting field line is defined by its polar coordinates $r$ and $\theta \cdot \chi$ is defined as the angle between the magnetic axis and the foot point of the field line on the surface of the star.

\subsection{Results of the modelling}

For both the curvature-radiation model and the plasmafrequency model we did not find any sets of values for the parameters to describe the frequency dependence of the profile widths correctly. The best set of parameters gave a reduced chisquare above 300 and above 15 for the curvature-radiation and the plasma-frequency model, respectively. In contrast, we found that the empirical model, which includes an extra parameter, 
Table 7. Parameters and their ranges of variation used to fit the curvature-radiation model (top), the plasma-frequency model (middle) and the empirical model (bottom).

\begin{tabular}{lll}
\hline \hline $\begin{array}{l}\text { Parameters for the } \\
\text { curvature radiation } \\
\text { model }\end{array}$ & lower limit & upper limit \\
\hline$\alpha$ & $0.1^{\circ}$ & \\
$\beta$ & $0.1^{\circ}$ & $6^{\circ}$ \\
$\gamma$ & 10 & 2000 \\
$\chi_{0}$ & $0.3^{\circ}$ & $0.92^{\circ}$ \\
$\chi_{\mathrm{w}}$ & $0.06^{\circ}$ & $0.57^{\circ}$ \\
\hline Parameters for the & 1 lower limit & upper limit \\
plasma-frequency & & \\
model & & \\
\hline$\alpha$ & $0.1^{\circ}$ & $6^{\circ}$ \\
$\beta$ & $0.1^{\circ}$ & $6^{\circ}$ \\
$\omega_{0}$ & $0.01 \mathrm{GHz}$ & $1000 \mathrm{GHz}$ \\
$\chi_{0}$ & $0.3^{\circ}$ & $0.92^{\circ}$ \\
$\chi_{\mathrm{w}}$ & $0.06^{\circ}$ & $0.57^{\circ}$ \\
\hline Parameters for the & $10 \mathrm{wer} \mathrm{limit}$ & upper limit \\
empirical model & & \\
\hline$\alpha$ & $0.1^{\circ}$ & $6^{\circ}$ \\
$\beta$ & $0.1^{\circ}$ & $6^{\circ}$ \\
$h_{0}$ & $10 \mathrm{~km}$ & $150 \mathrm{~km}$ \\
$\omega_{0}$ & $0.01 \mathrm{GHz}$ & $1000 \mathrm{GHz}$ \\
$\chi_{0}$ & $0.3^{\circ}$ & $0.92^{\circ}$ \\
$\chi_{\mathrm{w}}$ & $0.06^{\circ}$ & $0.57^{\circ}$ \\
\hline & & \\
\hline
\end{tabular}

described the data with a reduced chi-square ranging from 0.7 to 3 for many different values for the parameters. Characteristic to these sets of parameters were low values for $h_{0}$ and $\omega_{0}$, suggesting a minimum emission height ranging from 1 to $50 \mathrm{~km}$ above the stellar surface and a maximum emission height ranging from 10 to $100 \mathrm{~km}$ above the stellar surface, where the lowest values for the chi-square always correspond to the lowest emission heights. We did not allow emission heights below the stellar surface, but such values would give low values for the reduced chi-square as well. For comparison, we tried to obtain emission heights by modelling the emission for different values of the parameters $\alpha, \chi_{0}$ and $\chi_{\mathrm{w}}$ ( $\beta$ was kept equal to $\alpha$ ) and making no assumptions about the relationship between height and frequency of emission. For each frequency, the emission height was changed in such a way that the width of the profile from the observation would be equal to the width of the profile from the model. This always resulted in small emission height differences between frequencies, varying from a few kilometers to a few tens of kilometers. Also, for each set of parameters it was possible to choose values for $h_{0}$ and $\omega_{0}$ for which the empirical model would fit well to the relationship obtained between height and frequency of emission.

Based on these results we proceeded to make a more complex model, including two drift modes of sub-pulse emission superposed on low-intensity diffuse emission, assuming the empirical model for obtaining the emission height for each frequency. The parameters of this model are listed in Table 8 , where $R_{\star}$ is the radius of the pulsar, $D_{\mathrm{w}}$ is the angle between the foot points on the surface of the star of the two field lines for which the intensity from the diffuse emission has dropped to $60 \%$ of the maximum intensity, $D_{\mathrm{I}}$ is the intensity of the diffuse emission divided by the intensity of the sub-pulse emission at the center field line, $N_{\text {sub }}$ is the number of sub-pulses and $P_{3}$ is the rotation time of the carousel divided by $N_{\text {sub. }}$. The superscripts A and $\mathrm{B}$ refer to the value for that parameter in drift mode $\mathrm{A}$ or $\mathrm{B}$. Note that we only changed the values of $\chi_{0}$ and $P_{3}$ to describe
Table 8. Optimised parameter values in a full geometrical model for the emission of PSR B0031-07, describing two modes of drifting subpulses superposed on low-intensity diffuse emission, assuming a radiusto-frequency mapping based on the empirical model of Thorsett (1991).

\begin{tabular}{llll}
\hline \hline Parameter & Value & Parameter & Value \\
\hline$P_{1}$ & $0.9429 \mathrm{~s}$ & $\chi_{0}^{\mathrm{A}}$ & $0.85^{\circ}$ \\
$R_{\star}$ & $10 \mathrm{~km}$ & $\chi_{0}^{\mathrm{B}}$ & $0.81^{\circ}$ \\
$\alpha$ & $1.83^{\circ}$ & $\chi_{\mathrm{w}}$ & $0.13^{\circ}$ \\
$\beta$ & $1.83^{\circ}$ & $D_{\mathrm{w}}$ & $0.22^{\circ}$ \\
$h_{0}$ & $11 \mathrm{~km}$ & $D_{\mathrm{I}}$ & 0.001 \\
$\omega_{0}$ & $1.206 \mathrm{GHz}$ & $N_{\text {sub }}$ & 9 \\
$P_{3}^{\mathrm{A}}$ & $13 \mathrm{~s}$ & & \\
$P_{3}^{\mathrm{B}}$ & $7 \mathrm{~s}$ & & \\
\hline
\end{tabular}

a change in drift mode. The rotation time of the carousel and the number of sub-beams were derived from the observed vertical spacing between drift bands, assuming that the observed sub-pulses are not aliased. If we are in fact observing an alias, then the rotation time of the carousel as well as the number of sub-pulses become less. Table 8 also lists the values of these parameters that were obtained after fitting them to describe the aforementioned features of the observations. The values for $h_{0}$ and $\omega_{0}$ in Table 8 lead to emission heights ranging from $2.3 \mathrm{~km}$, at $4.85 \mathrm{GHz}$, to $13.6 \mathrm{~km}$ at $0.157 \mathrm{GHz}$ above the stellar surface. Regardless of the relationship between height and frequency of emission, these low emission heights are needed to obtain the frequency dependence of $P_{2}$, which depends on the fractional change of emission height. The value for $\chi_{0}^{A}$ corresponds to the last open field line, which for a small value of $\alpha$ is given by $\chi_{\text {lof }}=\sqrt{R_{\star} 2 \pi / P_{1} c}$.

Figure 5 shows a gray scale plot of the single pulses from the observations on the left and of the single pulses from the model on the right, for both the highest and smallest frequencies of the simultaneous observations. It demonstrates that the model can indeed reproduce both mode $\mathrm{A}$ and mode $\mathrm{B}$ drifts and also that the mode B drift disappears at high frequency. It also shows the change in profile width. We further calculated the widths of the average intensity profiles and the values for $P_{2}$ from the model for both drift modes at all frequencies. For each frequency, the signal-to-noise ratio was adjusted to be the same as the signal-tonoise ratio of the data. The widths of both the average intensity profiles and the average drift profiles from both the model and from the observations for drift mode $\mathrm{A}$ and $\mathrm{B}$ at each frequency are shown in Fig. 6. The average fractional drift intensity from both the model and the observations for drift mode A and B at each frequency are shown in Fig. 7. Figure 8 shows the values of $P_{2}$ from both the model and from the observations at each frequency. Since the difference in $P_{2}$ between driftmodes due to their different values for $\chi_{0}$ is far less than the error on the measured values for $P_{2}$, the values of $P_{2}$ for drift mode $\mathrm{A}$ and drift mode $B$ were averaged together. Images of the model can be seen in Fig. 9.

\section{Discussion}

Figure 2 shows that there is only one polarisation-mode present whenever the pulsar is in drift mode A, whereas drift mode B shows two orthogonal modes of polarisation, as has been reported by Smits et al. (2005). They also report that in their $325 \mathrm{MHz}$ observations the mode-A profile shows a double component, indicating that the geometry of the emission region during this drift mode is such that over a few degrees of pulse phase, one sees emission from a region between the magnetic axis and 

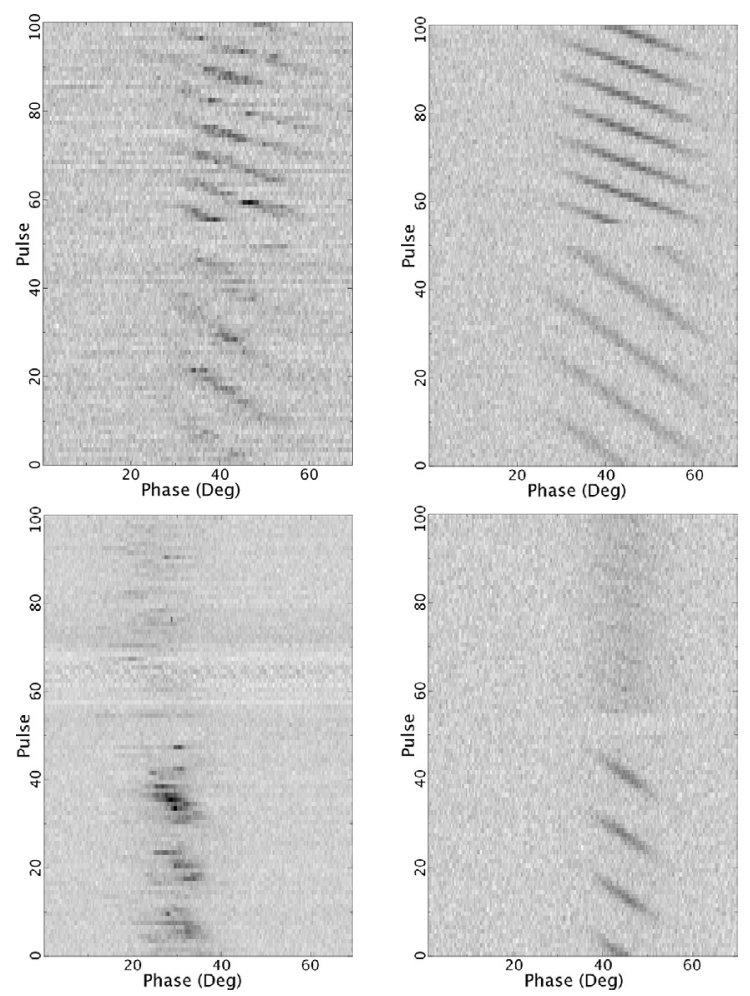

Fig. 5. Gray scale plots of single pulses at two frequencies from the simultaneous observations (left panels) and from the model (right pan$e l s$ ). The upper plots are at $243 \mathrm{MHz}$ and the bottom plots are at $4.85 \mathrm{GHz}$. The first 50 pulses are in drift mode A, the following 5 pulses are nulls and the remaining pulses are in drift mode B. The pulses in the model were aligned with the pulses from the 243-MHz observation. This results in the offset between single pulses from the observation and from the model at $4.85 \mathrm{GHz}$. There is some interference visible in the pulses 60 to 68 of the observation at $4.85 \mathrm{GHz}$.

the maximally emitting field lines. This is confirmed by the average intensity profiles from Izvekova et al. (1993), where a double component becomes visible at $62 \mathrm{MHz}$. However, our current observations only show a hint of a double component in the mode-A profiles. An explanation for this might be that the peak of the mode-A emission zone during the present observations lies slightly closer to the magnetic axis than the peak of the mode-A emission zone during the observations from Smits et al. (2005). This makes it possible that no emission was detected from the region between the magnetic axis and the maximally emitting field line in the low frequency observations presented here. The position angles in Fig. 2 reveal that orthogonal polarisation mode jumps occur at all frequencies. However, in some cases the jump is not instantaneous, but rather there is a resolved transition, as can be seen at $243 \mathrm{MHz}$. This is not consistent with a mode jump resulting from two competing uncorrelated orthogonal modes of polarisation. Also, the resolved transition, present in the $607-\mathrm{MHz}$ observation is in the opposite direction of the transition that is visible at other frequencies. This difference is not a result from an incorrect polarisation calibration, as it can be seen that the underlying position angle sweep is identical to that at other frequencies. A brief study of smaller sections of the $607-\mathrm{MHz}$ observation revealed that the average position angle sweep of about 1000 pulses can contain an orthogonal jump in which the position angle either increases within a few degrees of pulsar phase, decreases within a few degrees of pulsar phase, or changes within a timescale smaller than the timeresolution.
Further, Fig. 2 shows that the average intensity profiles of PSR B0031-07 shift to earlier phase with increasing frequency. This can also be seen in the average profiles from Izvekova et al. (1993). They suggest that the shift can be removed by the adoption of a higher value of dispersion measure. Since Izvekova et al. have determined the dispersion measure by aligning the sub-pulses in the center of the profile, which is similar to the method of alignment used for the present observations, we briefly check if both the shift in their observations and the shift in the current observations could be due to twisted magnetic field lines. Since the sub-beams follow the magnetic field lines, the drift phase of the sub-pulses will differ between frequencies. Thus by "artificially" aligning the sub-pulses between frequencies, a shift will be introduced between the average intensity profiles. To obtain the observed shift, the magnetic field lines need to be twisted around the magnetic axis by approximately $20^{\circ}$ between the emission altitudes observed at $157 \mathrm{MHz}$ and $4.85 \mathrm{GHz}$. For the present model, this implies a twist of $2^{\circ}$ per kilometer altitude. We can estimate the twist of the field lines due to a current of charged particles with a density equal to the Goldreich-Julian density, streaming away from the pulsar surface at the speed of light and filling the entire polar cap. The pulse phase shift $\Delta \phi$ over a height difference $\Delta h$ is then estimated as

$\frac{\Delta \phi}{\Delta h}=\frac{B_{\phi}}{R_{\mathrm{pc}} B_{z}}=\frac{2 \pi}{P_{1} c}$

where $B_{\phi}$ and $B_{z}$ are the $\phi$ and $z$ components of the magnetic field, $R_{\mathrm{pc}}$ is the radius of the polar cap and $P_{1}$ is the rotation period of the pulsar. For this pulsar this leads to a twist of only $0.0013^{\circ}$ per kilometer altitude. To overcome this difference of a factor of 1000 , one needs to assume either unrealistically large current densities or much greater emission height differences.

From Fig. 3 it becomes evident that the mode-B drift is seen less frequently towards higher frequencies. Interestingly, even though the occurrence decreases with increasing frequency, the fractional drift intensity itself of this drift mode does not drop until $4.85 \mathrm{GHz}$ and even at this frequency it can still be seen occasionally. This is in contrast with the results from Smits et al. (2005) who did not see any mode-B drift sequences in 2700 pulses at $4.85 \mathrm{GHz}$, which were observed simultaneously at $325 \mathrm{MHz}$, nor in 5350 pulses at $1.41 \mathrm{GHz}$, which were not observed simultaneously at any other frequency. Of course, in the absence of a simultaneous observation at low frequency, a mode-B drift at high frequency might remain undetected. Also, due to the infrequent occurrence of the mode-B drift at $4.85 \mathrm{GHz}$, it is possible that Smits et al. did not detect this drift mode at $4.85 \mathrm{GHz}$ because it did not occur during their observation. Alternatively, the location of the mode-B emission during the present observation might differ from the location of the mode-B emission during the observations from Smits et al. (2005). The fact that a fractional drift intensity can only be detected sporadically for mode B at $4.85 \mathrm{GHz}$, does suggest that the location of the mode-B emission can slightly change on timescales of a few minutes. Despite these changes over small timescales, the average fractional drift intensities that are shown in Table 4 represent how the line of sight intersection with the emission region changes over frequency. However, it should be noted that residual interference and variations in the sub-pulse intensity within a drift sequence can affect these values. These effects result in the large errors listed in Table 4. Still, the values in Table 4 are consistent with the suggestion that at all frequencies a clear mode-A drift can be detected, whereas the mode-B drift becomes harder to detect towards higher frequencies. This 

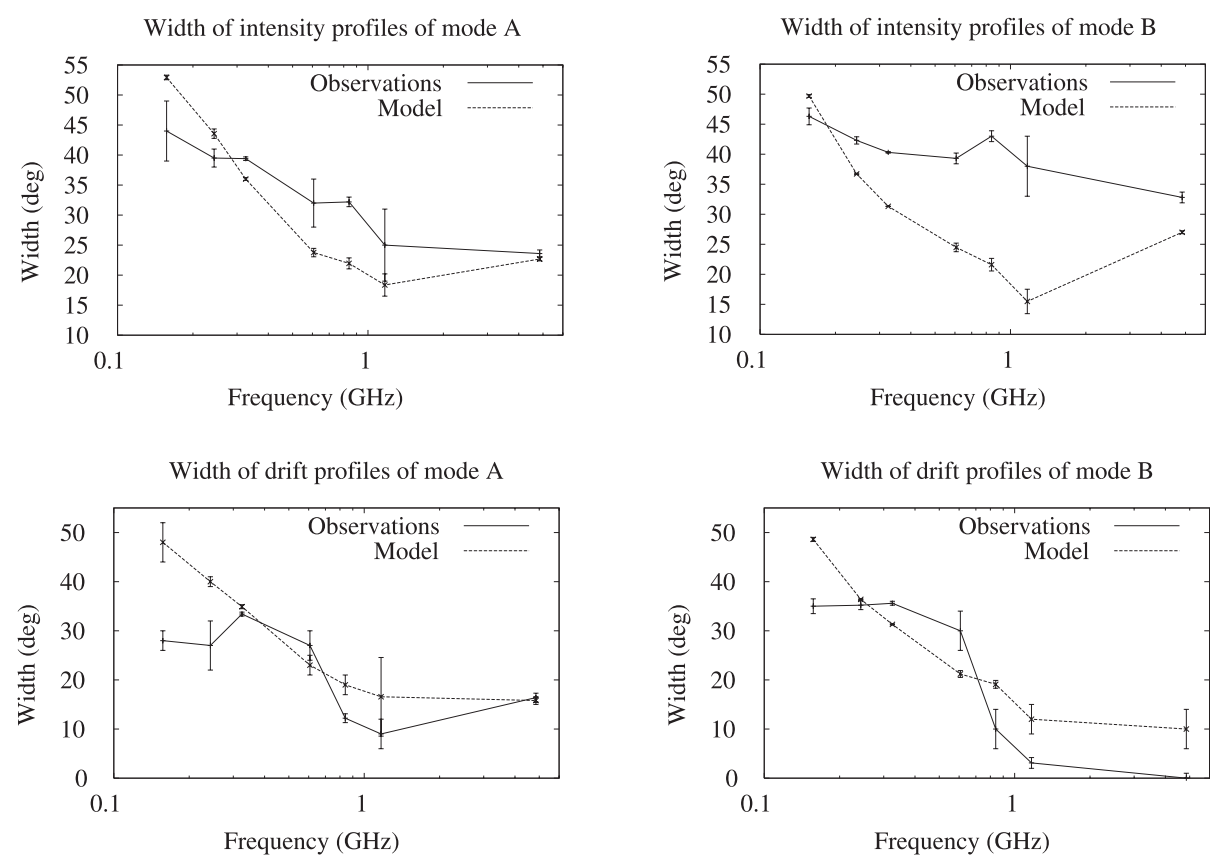

Fig. 6. Widths of the average intensity profiles (upper plots) and average drift intensities (lower plots) from the observations (solid line) and from the model (dashed line), for drift modes A (left plots) and B (right plots) at each frequency.
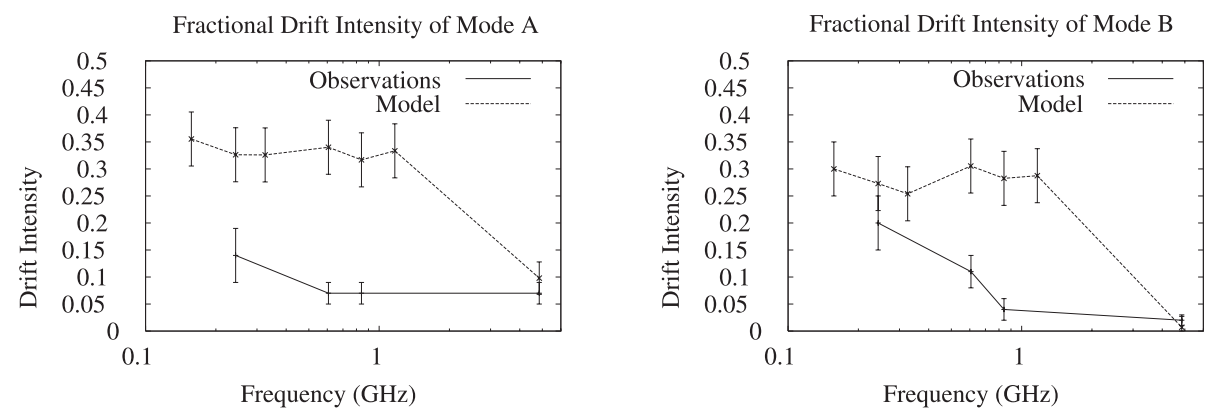

Fig. 7. Average fractional drift intensity from the observations (solid line) and from the model (dashed line), for drift modes A (left plot) and B (right plot).

can also be seen in Table 5, where the widths of the average mode-B drift profiles decrease with increasing frequency. The widths of the average mode-A drift profiles can also be seen to decrease with increasing frequency, but even at the highest frequency, the profile is still broad enough to allow detection.

Figure 5 shows that the model can reproduce the single pulses of both drift mode A and B, except for the shift in arrival time between the single pulses at low and high frequencies. This shift can also be seen in the average profiles of Fig. 2. The change of the width of the average intensity profiles with frequency can be seen in Fig. 6. It is clear that the widths of the intensity profiles from the model have a steeper spectrum than those widths from the observations. This steep spectrum is a consequence of the change in height by a factor of 2 , which is required to obtain an observable change in $P_{2}$. At $4.85 \mathrm{GHz}$, the width from the model becomes broader again due to the increased intensity of the broad non-drifting emission with respect to the emission associated with the drifting sub-pulses. Figure 7 reveals that the average fractional drift intensity from the model is overall much higher than the average fractional drift intensity from the observations. This is as expected, since the model does not include pulse to pulse intensity fluctuations, which will lower the fractional drift intensity. Alternatively, the different spectra of the drifting and non-drifting emission might be due to a different emission process rather than a different geometry. However, from the results presented here, we feel that it is not necessary to assume different emission processes. The model does reproduce the disappearing of drift mode $\mathrm{B}$ at $4.85 \mathrm{GHz}$, as was already seen in Fig. 5. Figure 8 shows the change of $P_{2}$ over frequency from the observations and from the model. At low frequency the change of $P_{2}$ from the observation and from the model are similar. However at high frequencies, the values of $P_{2}$ from the observations are significantly lower than the values of $P_{2}$ from the model. A steeper spectrum of $P_{2}$ in the model can be obtained by making the emission height difference between the high and low frequencies larger. However, this would result in a steeper spectrum for the width of the average intensity profiles, which does not match the observations. A possible explanation might be that the method used to measure $P_{2}$ is influenced by the non-drifting emission, which we suggest is present along with the drifting sub-pulses. The value of $P_{2}$ is linearly related to the sub-pulse phase drift, which is measured by cross-correlating consecutive pulses. In the presence of non-drifting emission, this cross-correlation not only peaks at the sub-pulse phase drift, but it will also have a broad peak at zero phase shift. When the intensity of the non-drifting emission becomes strong with respect to the intensity of the drifting emission, which happens at high frequencies, both peaks in the average cross-correlation will merge 


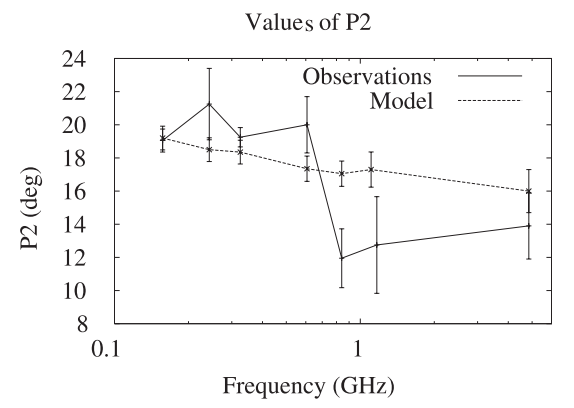

Fig. 8. Value of $P_{2}$ from the observations (solid) and from the model (dashed) at each frequency. Note that the presence of non-drifting emission affects the method used to measure $P_{2}$. (See discussion for more details.)

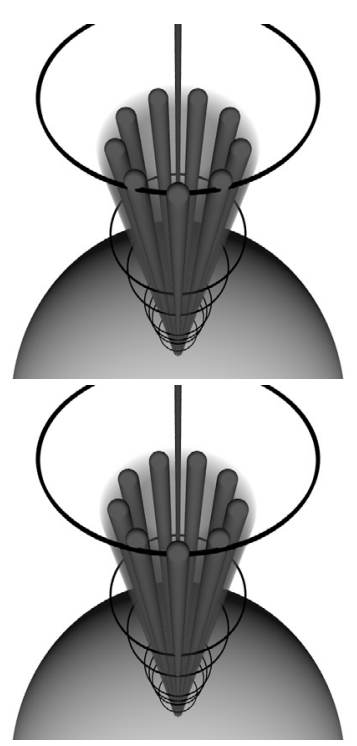

Fig. 9. Two close ups of the model of the emission zone of PSR B0031-07. The vertical line is the rotation axis of the pulsar. The 7 circles indicate the line of sight trajectories corresponding to the 7 observed frequencies. The emission zone consists of 9 sub-beams surrounded by diffuse emission, shown as semi-transparent. Both images are to scale. The top image shows the location of the sub-beams during mode-A drift. The bottom image shows the location of the sub-beams during mode-B drift, which lie slightly closer to the magnetic axis.

into one peak. This results in a lower value for the sub-pulse phase drift and thus also for $P_{2}$. At low frequencies we do not expect the value for $P_{2}$ to be influenced by the non-drifting emission as it is very weak compared to the drifting emission. And indeed, our present values of $P_{2}$ at low frequencies are similar to those from Izvekova et al. (1993), or those from Fig. 9 from Bartel et al. (1980), who used auto-correlation functions to directly measure $P_{2}$. Finally, Fig. 9 show the actual geometry and emission heights of the emission zone of PSR B0031-07, as they result from the model. The lowest and highest circles lie at heights of $2.3 \mathrm{~km}$ and $13.6 \mathrm{~km}$ from the surface of the star, respectively. Figure 9 shows that the change in the location of the sub-beams between mode-A and mode-B drift is very small.

\section{Conclusions}

We have shown the results from an analysis of a simultaneous multifrequency observation of PSR B0031-07. We have included two non-simultaneous observations to obtain a total of 7 different frequencies. From these observations we first determined the drift mode of each drift sequence at each frequency. Contrary to what was expected, we found multiple detections of mode-B drift at $4.85 \mathrm{GHz}$. We then determined the position angle sweep, the width of the average intensity profile, the width of the average drift profile and $P_{2}$ for each drift mode at each frequency. We then tried to fit three emission models to the widths of the average intensity profiles to ultimately find a model that describes all the observed features. We found that the models based on curvature radiation and plasma-frequency emission could not reproduce the frequency dependence of the widths. However, the model based on an empirical relationship between the height and frequency of emission, which includes an extra parameter, could reproduce the frequency dependence of the widths for many different values of the parameters. We therefore improved the latter model to reproduce two drift modes of the single pulse emission.

We can summarize the features of the geometrical model of PSR B0031-07 that is presented here as follows.

- The model reproduces to great extent the position angle sweep (without the orthogonal mode jumps) and the frequency dependences of the width of the average intensity profiles, the width of the average drift profiles, the fractional drift intensity and $P_{2}$, for drift modes $\mathrm{A}$ and $\mathrm{B}$ of the single pulses of PSR B0031-07. The largest deviations are found in the average intensity profiles.

- The emission heights are very low. The high frequency emission comes from a region just above the surface of the star. The low frequency emission comes from a region about 10 kilometers higher than the high frequency emission.

- The parameters $\alpha$ and $\beta$ are approximately the same and depending on the actual emission height, around $2^{\circ}$ to $4^{\circ}$.

- The emission is centered around, or close to the last open field lines.

- The emission from drift mode B comes from a region just slightly closer to the magnetic axis than the emission from drift mode A.

- Along with the drifting sub-pulses there is non-drifting emission in the single pulses that becomes more significant towards higher frequencies. This non-drifting emission might provide a hint as to why not all pulsars show clear drifting sub-pulses.

- Assuming that the observed drift speeds of the sub-pulses are not aliased, the number of sub-beams is around 9.

The model results in very low emission altitudes, ranging from 2.3 to $13.6 \mathrm{~km}$ above the surface of the star. This is in strong contrast with other emission heights that have been measured for pulsars, which are typically some 10 to $1000 \mathrm{~km}$ (e.g. von Hoensbroech \& Xilouris 1997; Gangadhara \& Gupta 2001; Mitra \& Rankin 2002; Mitra \& Li 2004). However, by improving the model, the emission heights can become higher. The model might be improved by assuming a more realistic particle-density distribution near the polar cap, resulting in variable emission heights at a fixed emission frequency. This can also improve the plasma-frequency model. Also, the emission altitude of mode A and mode B need not be the same, as has been assumed here. Karastergiou for his help on data alignment and an anonymous referee for his/her usefull comments. This paper is based on observations with the $100-\mathrm{m}$ telescope of the MPIfR (Max-Planck-Institut für Radioastronomie) at Effelsberg, the Westerbork Synthesis Radio Telescope and the Giant Metrewave Radio 
Telescope and we would like to thank the technical staff and scientists who have been responsible for making these observations possible.

\section{References}

Bartel, N., Sieber, W., \& Graham, D. A. 1980, A\&A, 87, 282

Edwards, R. T., \& Stappers, B. W. 2004, A\&A, 421, 681

Gangadhara, R. T. 2004, ApJ, 609, 335

Gangadhara, R. T., \& Gupta, Y. 2001, ApJ, 555, 31

Gupta, Y., et al. 2000, in Pulsar Astronomy - 2000 and Beyond, IAU, 177

Hoensbroech, A. G. 1999, Ph.D. Thesis

Huguenin, G. R., Taylor, J. H., \& Troland, T. H. 1970, ApJ, 162, 727

Izvekova, V. A., Kuzmin, A. D., Lyne, A. G., Shitov, Y. P., \& Smith, F. G. 1993, MNRAS, 261, 865

Jackson, J. D. 1999, 3rd edn.

Joshi, B. C., \& Vivekanand, M. 2000, MNRAS, 316, 716

Krishnamohan, S. 1980, MNRAS, 191, 237

Kuzmin, A. D., Malofeev, V. M., Izvekova, V. A., Sieber, W., \& Wielebinski, R. 1986, A\&A, 161, 183
Mitra, D., Gupta, Y., \& Kudale, S. 2005, Polarization Calibration of the Phased Array Mode of the GMRT, URSI GA 2005, Commission J03a

Mitra, D., \& Li, X. H. 2004, A\&A, 421, 215

Mitra, D., \& Rankin, J. M. 2002, ApJ, 577, 322

Radhakrishnan, V., \& Cooke, D. J. 1969, Astrophys. Lett., 3, 225

Ruderman, M. A., \& Sutherland, P. G. 1975, ApJ, 196, 51

Smits, J. M., Mitra, D., \& Kuijpers, J. 2005, A\&A, 440, 683

Swarup, G., Ananthakrishnan, S., Kapahi, V. K., et al. 1991, Current Sci., 60, 95

Taylor, J. H., Manchester, R. N., \& Lyne, A. G. 1993, ApJS, 88, 529

Thorsett, S. E. 1991, ApJ, 377, 263

Vivekanand, M. 1995, MNRAS, 274, 785

Vivekanand, M., \& Joshi, B. C. 1997, ApJ, 477, 431

Vivekanand, M., \& Joshi, B. C. 1999, ApJ, 515, 398

Voûte, J. L. L., Kouwenhoven, M. L. A., van Haren, P. C., et al. 2002, A\&A, 385, 733

von Hoensbroech, A., \& Xilouris, K. M. 1997, A\&A, 324, 981

Wright, G. A. E. 1981, MNRAS, 196, 153

Wright, G. A. E., \& Fowler, L. A. 1981, in Pulsars: 13 Years of Research on Neutron Stars, IAU Symp., 95, 211 